\title{
Study of electrical circuits using Runge Kutta method of order 4
}

Malarvizhi $\mathrm{M}^{1}$, Karunanithi $\mathrm{S}^{2}$

Received: 21 October 2021/ Accepted: 08 November 2021/ Published online: 21 December 2021

CSacred Heart Research Publications 2017

\begin{abstract}
In this paper, Runge Kutta method of order 4 is used to study the electrical circuits designs through past, intermediate and present voltages. When integrating differential equations with Runge Kutta method of order 4, a constant step size $(h)$ is used until a testing procedure confirms that the discontinuity occurs in the present integration interval. This step size function calculations would take place at the end of the functional calculations, but before the dependent variables were updated. Runge Kutta methods along with convolution are given by array interpretation (Butcher matrix) representation, this leads to identify the equilibrium state. The input parameters indicate the voltage coefficient controlled by current sources and measures it a random periodic time. The output parameters provide stable independent values and calculated from past voltage and current values. Finally solutions are compared with exact values and RK method of order 4 along with Heun, Midpoint and Taylors's method with various $h$ values.
\end{abstract}

Key Words: Runge Kutta method of order 4, step size, past, intermediate and present voltages, electrical circuits.

AMS Classification: 34B15, 34B16.

\footnotetext{
${ }^{1}$ PG \& Research Department of Mathematics, Government Thirumagal Mills College, Tamil Nadu, India. Email: malar2308@gmail.com

${ }^{2}$ PG \& Research Department of Mathematics, Government Thirumagal Mills College, Tamil Nadu, India. Email: kap232008@gmail.com
} 


\section{Introduction}

Numerous branches of economics, science, engineering and technology are commonly contain numerical methods and analysis. Runge Kutta Method of order 4 is reasonably simple and robust and is used to analyze the transient behaviors of RLC circuit taken into consideration of higher order accuracy in multi-stage. It involves slope calculations at multiple steps between the current and the consecutive discrete time values combined with an intelligent adaptive step-size routine [4]. RK method of order 4 preserves stability, provided that the step size $h$ does not become too large $[7,11]$. In the general observation, higher order RK methods allows us to increase / decrease the step size and obtaining good accuracy but the stability of the algorithms establishes limits to the value of $h$. This method avoids the higher order derivatives of the unknown function $y$ and obtained the higher order accuracy [18] because of its computation potential, accuracy, flexibility and the possibility of changing the integration step. Large scale parallel processing system without redesigning the structure is processed by Runge Kutta method of order 4 with small $h$ values [3, 14]. An efficient implementation and designing the circuits are evaluated and analyzed the scalability and throughput $[5,16]$.

The Runge Kutta technique is a one-step method with several stages whose order is determined by the number of stages $[1,17]$. This technique and approach is used to solve differential equations of various types, including explicit, implicit, partial, and delay differential equations $[2,10]$. This method played a significant role in the study of explicit and implicit iterative strategies for solving ODEs with temporal dissipation. Higher order stability preserving Runge Kutta discretizations were created for use with semi-discrete method of approximations of hyperbolic partial differential equations, and have been found to be useful in a diverse of other situations [6]. Because hyperbolic problems and its issues are often leads discontinuous solutions and the nonlinear stability features are almost critical [9, 15].

When utilizing a linearly stable, high-order approach that lacks the strong stability feature and numerical data showed that oscillations can arise [8, 13]. For a nonlinear ODE, any Runge Kutta technique of fifth order or above will have at least one negative coefficient. As a result, we recognize that negative coefficients must be addressed when using Runge Kutta methods to solve nonlinear ODEs [12]. 
This paper is organized as follows. Section II focused the convolution of Runge Kutta method of order 4. Equilibrium state and array interpretation (Butcher matrix) representation of RK method of order 4 along with the convolution of past, intermediate and present voltages are studied in this section. In section III, the input parameters indicate the voltage coefficient which is controlled by current sources and measures in a random periodical time. The output parameters provide stable independent values and calculated from past voltage and current values. Section IV presents the implementation of numerical integration for nonlinear capacitors. Comparing exact values with RK method of order 4, Heun, Midpoint and Taylors's method with various $h$ values are calculated. Finally section V concludes the paper.

\section{Convolution and Runge Kutta method of order 4}

Consider the following first order linear differential equation,

$$
y^{\prime}=f(t, y)
$$

Runge Kutta methods along with convolution are given by

$$
\begin{gathered}
Y_{i}=y_{n}+h \sum_{j=1}^{s} a_{i j} * f\left(t_{n}+c_{j} h, Y_{j}\right) \quad(i=0,1, \ldots, s) \\
y_{n+1}=y_{n}+h \sum_{j=1}^{s} b_{i} * f\left(t_{n}+c_{i} h, Y_{i}\right)
\end{gathered}
$$

(1) is given by the array interpretation (Butcher matrix) representation and this type arrangement is shown in table 1 and is explicit when upper triangular coefficients $a_{i j}(i \leq j)$ are zero and pass through towards equilibrium state and implicit except these conditions (i.e., $a_{i j}(i \leq j)$ are non zero and does not pass through equilibrium state.

\begin{tabular}{c|cccc}
$c_{1}$ & $a_{11}$ & $a_{12}$ & $\ldots$ & $a_{1 s}$ \\
$c_{2}$ & $a_{21}$ & $a_{22}$ & $\ldots$ & $a_{2 s}$ \\
$\vdots$ & $\vdots$ & $\vdots$ & $\ddots$ & $\vdots$ \\
$c_{s}$ & $a_{s 1}$ & $a_{s 2}$ & $\ldots$ & $a_{s s}$ \\
\hline
\end{tabular}


Table 1: Array interpretation (Butcher matrix) representation of equation 1

Assume that $m_{i j}=a_{i j}, m_{10}=a_{20}=0, \ldots, m_{i j}=a_{(i-1)(j-1)}$.

$$
\begin{array}{r}
Y\left(t_{a}\right)-y\left(t_{n}\right)-h m_{10} f\left(t_{n}\right)-h m_{11} f\left(t_{a}\right)-h m_{12} f\left(t_{n+1}\right)=0 \\
y\left(t_{n+1}\right)-y\left(t_{n}\right)-h m_{20} * f\left(t_{n}\right)-h m_{21} * f\left(t_{a}\right)-h m_{22} * f\left(t_{n+1}\right)=0
\end{array}
$$

where $t_{n}, t_{a}$ and $t_{n+1}$ indicates past, intermediate and present time domain.

\section{Equivalent models for a linear element -Linear device}

Applying (2) to the following equation for a linear capacitor $(C)$ and voltage $v_{c}$ :

$$
i(t)=C \frac{d v_{c}}{d t}
$$

And we get

$$
\begin{aligned}
i_{a} & =\frac{\left(m_{12}-m_{22}\right) v_{n}+m_{22} v_{a}-m_{22} v_{n+1}-\frac{h}{c}\left(m_{10} m_{22}-m_{12} m_{20}\right) i_{n}}{\frac{h}{c}\left(m_{11} m_{22}-m_{12} m_{21}\right)} \\
i_{n+1} & =\frac{\left(-m_{11}+m_{21}\right) v_{n}-m_{21} v_{a}+m_{11} v_{n+1}+\frac{h}{c}\left(m_{10} m_{22}-m_{11} m_{20}\right) i_{n}}{\frac{h}{c}\left(m_{11} m_{22}-m_{12} m_{21}\right)}
\end{aligned}
$$

where $v_{n}, v_{a}$ and $v_{n+1}$ indicate a past, intermediate and present voltages. $i_{n}$ represent the final current value at time $t$. The equation for the simulation is given by

$$
\left[\begin{array}{rrrr}
B & -B & A & -A \\
-B & B & -A & A \\
E & -E & D & -D \\
-E & E & -D & D
\end{array}\right]\left[\begin{array}{c}
v_{1, n+1} \\
v_{2, n+1} \\
v_{1, a} \\
v_{2, a}
\end{array}\right]=\left[\begin{array}{r}
-P \\
P \\
-F \\
F
\end{array}\right]
$$

where $v_{1, a}$ and $v_{2, a}$ are the voltages in the intermediate time $t_{a} \cdot v_{1, n+1}$ and $v_{2, n+1}$ are the voltages at a present time $t_{n+1}$ and $t_{n+1}$. The input parameters $A$ and $E$ indicates the 
coefficients of the voltage controlled by current sources. In particular $A$ is controlled by $v_{c a}$ and $E$ is controlled by $v_{c n+1}$. Further $A$ and $E$ are defined like:

$$
A=\frac{-m_{21}}{\frac{h}{c}\left(m_{11} m_{22}-m_{12} m_{21}\right)}, \quad E=\frac{-m_{12}}{\frac{h}{c}\left(m_{11} m_{22}-m_{12} m_{21}\right)}
$$

The input parameters $B$ and $D$ indicates the coefficients of the voltage in a particular random periodic time. Further $A$ and $E$ are defined like:

$$
B=\frac{m_{11}}{\frac{h}{c}\left(m_{11} m_{22}-m_{12} m_{21}\right)}, \quad D=\frac{m_{22}}{\frac{h}{c}\left(m_{11} m_{22}-m_{12} m_{21}\right)}
$$

The output parameters $P$ and $F$ are stable independent values and calculated from past voltage and current values. Further $P$ and $F$ are defined like:

$$
\begin{gathered}
P=\frac{\left(-m_{11}+m_{21}\right) v_{c_{n}}+\frac{h}{c}\left(m_{10} m_{20}-m_{12} m_{21}\right) i_{c_{n}}}{\frac{h}{c}\left(m_{11} m_{22}-m_{12} m_{21}\right)} \\
F=\frac{\left(m_{12}-m_{22}\right) v_{c_{n}}+\frac{h}{c}\left(m_{10} m_{22}-m_{12} m_{20}\right) i_{c_{n}}}{\frac{h}{c}\left(m_{11} m_{22}-m_{12} m_{21}\right)}
\end{gathered}
$$

Here $i_{c_{n}}$ represents the current that pass through the capacitor. The similar equivalent circuits can be compared with the Runge Kutta method of order 4 and, moreover, to ensure the stability conditions of the variables are verified at random periodical time.

\section{Implementation of numerical integration for nonlinear capacitors}

This implementation consists of resistors, diodes and voltages. A diode model consists of a contact resistance $R_{s}$, a nonlinear conductor $g(v)$ and a nonlinear capacitor $q(v)$. The relationship between the nonlinear capacitor $q(v)$ with the voltage be like:

$$
q(v)=T_{t} I_{s}\left(\exp \left(\frac{v}{N V_{t}}\right)-1\right)
$$

where $T_{t}, I_{s}$ and $N$ indicates run time, a saturation current and coefficient of emission. Applying higher iterations to $i_{c_{n}}$, 


$$
\begin{aligned}
i_{c n}^{(j+1)}=\frac{m_{22}}{h \alpha}\{q & \left.\left(v_{a}^{(j)}\right)+C_{a}^{(j)}\left(v_{a}^{(j+1)}-v_{a}^{(j)}\right)\right\}-\frac{m_{12}}{h \alpha}\left\{q\left(v_{n+1}^{(j)}\right)+C_{n+1}^{(j)}\left(v_{n+1}^{(j+1)}-v_{n+1}^{(j)}\right)\right\} \\
& +\frac{1}{h \alpha}\left\{\left(m_{12}-m_{22}\right) q\left(v_{(n)}\right)-h\left(m_{10} m_{22}-m_{12} m_{20}\right) C_{n}\right\} \\
i_{c n+1}^{(j+1)}=\frac{m_{22}}{h \alpha}\{q & \left.\left(v_{n+1}^{(j)}\right)+C_{n+1}^{(j)}\left(v_{n+1}^{(j+1)}-v_{n+1}^{(j)}\right)\right\}-\frac{m_{12}}{h \alpha}\left\{q\left(v_{a}^{(j)}\right)+C_{a}^{(j)}\left(v_{a}^{(j+1)}-v_{a}^{(j)}\right)\right\} \\
& +\frac{1}{h \alpha}\left\{\left(m_{21}-m_{11}\right) q\left(v_{(n)}\right)+h\left(m_{10} m_{21}-m_{11} m_{20}\right) C_{n}\right\}
\end{aligned}
$$

where $j$ represents the number of iterations. Parameters $C_{a}^{(j)}, C_{n+1}^{(j)}, C_{n}$ and $\alpha$ in (6) are given by

$$
\begin{array}{lr}
C_{a}^{(j)}=\left.\frac{\partial q(v)}{\partial v}\right|_{v=v_{a}^{(j)}} & C_{n+1}^{(j)}=\left.\frac{\partial q(v)}{\partial v}\right|_{v=v_{n+1}^{(j)}} \\
C_{n}=\left.\frac{\partial q(v)}{\partial v}\right|_{v=v_{n}} & \alpha=m_{11} m_{22}-m_{12} m_{21}
\end{array}
$$

Equation (6) is rewritten as a simplified form as:

$$
\begin{aligned}
& i_{c a}^{(j+1)}=G_{c a}^{(j)} v_{a}^{(j+1)}+g_{c n+1}^{(j)} v_{n+1}^{(j+1)}+I_{c a}^{(j)} \\
& i_{c n+1}^{(j+1)}=G_{c n+1}^{(j)} v_{n+1}^{(j+1)}+g_{c a}^{(j)} v_{a}^{(j+1)}+I_{c n+1}^{(j)}
\end{aligned}
$$

where $G_{c a}^{(j)}$ and $G_{c n+1}^{(j)}$ are conductance's and are defined by

$$
G_{c a}^{(j)}=\frac{m_{22}}{h \alpha} C_{a}^{(j)} \quad G_{c n+1}^{(j)}=\frac{m_{11}}{h \alpha} C_{n+1}^{(j)}
$$

$g_{c n+1}^{(j)}$ and $g_{c a}^{(j)}$ in (7) shows the coefficients of voltage. Simultaneously the coefficient of $g_{c n+1}^{(j)}$ is connected to the equivalent circuit at $t_{a}$ and controlled by voltage at $t_{n+1}$. Parameter $g_{c a}^{(j)}$ is connected to the equivalent circuit at $t_{n+1}$ and controlled by voltage at $t_{a}$ and defined as

$$
g_{c n+1}^{(j)}=-\frac{m_{12}}{h \alpha} C_{n+1}^{(j)} \quad g_{c a}^{(j)}=-\frac{m_{21}}{h \alpha} C_{a}^{(j)}
$$

$I_{c a}^{k}$ and $I_{c n+1}^{k}$ in (7) are defined like 


$$
\begin{gathered}
I_{c a}^{(j)}=I_{c 1}^{(j)}+i_{c 1}^{(j)}+I_{c 1 n} \\
I_{c n+1}^{(j)}=I_{c 2}^{(j)}+i_{c 2}^{(j)}+I_{c 2 n}
\end{gathered}
$$

where each term is calculated by

$$
\begin{gathered}
I_{c 1}^{(j)}=\frac{m_{22}}{h \alpha}\left[q\left(v_{a}^{(j)}\right)+T_{t} I_{s}-C_{a}^{(j)} v_{a}^{(j)}\right] \\
I_{c 2}^{(j)}=\frac{m_{11}}{h \alpha}\left[q\left(v_{n+1}^{(j)}\right)+T_{t} I_{s}-C_{n+1}^{(j)} v_{n+1}^{(j)}\right] \\
i_{c 1}^{(j)}=\frac{m_{12}}{h \alpha}\left[C_{n+1}^{(j)} v_{n+1}^{(j)}-q\left(v_{n+1}^{(j)}\right)-T_{t} I_{s}\right] \\
i_{c 2}^{(j)}=\frac{m_{21}}{h \alpha}\left[C_{a}^{(j)} v_{a}^{(j)}-q\left(v_{a}^{(j)}\right)-T_{t} I_{s}\right] \\
I_{c 1 n}=\frac{T_{t} I_{s}\left(m_{12}-m_{22}\right)}{h \alpha}+\frac{T_{t} I_{s}}{h \alpha}\left[\left\{\left(m_{12}-m_{22}\right)-h\left(m_{10} m_{22}-m_{12} m_{20}\right)\right\} q\left(v_{n}\right)+T_{t} I_{s}-1\right] \\
I_{c 2 n}=\frac{T_{t} I_{s}\left(m_{21}-m_{11}\right)}{h \alpha}+\frac{T_{t} I_{s}}{h \alpha}\left[\left\{\left(m_{21}-m_{11}\right)-h\left(m_{10} m_{21}-m_{11} m_{20}\right)\right\} q\left(v_{n}\right)+T_{t} I_{s}-1\right]
\end{gathered}
$$

The nonlinear physical phenomenon is linearized by applying Newton's methodology on $t_{a}$ and $t_{n+1}$ both are associated intermediately. Therefore, the equivalent nonlinear electrical model consists of $G_{g a}^{(j)}$ and $G_{g n+1}^{(j)}$ (conductance) and the parallel current sources $I_{g a}^{(j)}$ and $I_{g n+1}^{(j)}$ which are defined as:

$$
\begin{array}{ll}
G_{g a}^{(j)}=\left.\frac{\partial g(v)}{\partial v}\right|_{v=v_{a}^{(j)}} & G_{n+1}^{(j)}=\left.\frac{\partial g(v)}{\partial v}\right|_{v=v_{n+1}^{(j)}} \\
I_{g a}^{(j)}=g\left(v_{a}^{(j)}\right)-G_{g a}^{(j)} v_{a}^{(j)} & I_{g n+1}^{(j)}=g\left(v_{n+1}^{(j)}\right)-G_{g n+1}^{(j)} v_{n+1}^{(j)}
\end{array}
$$

Homogenous circuit in $(j+1)^{\text {th }}$ Newton's iteration and $(n+1)^{\text {th }}$ time domain are written like,

$$
\begin{array}{cc}
G_{d 1}^{(j)}=G_{g a}^{(j)}+G_{c a}^{(j)} & G_{d 2}^{(j)}=G_{g n+1}^{(j)}+G_{c n+1}^{(j)} \\
I_{d 1}^{(j)}=I_{g a}^{(j)}+I_{c a}^{(j)} & I_{d 2}^{(j)}=I_{g n+1}^{(j)}+I_{c n+1}^{(j)}
\end{array}
$$


The dimension of equivalent circuits becomes Runge Kutta method of order 4. The voltage controlled by the current $i_{C E}$ is controlled by $v_{B C}$ and $v_{B E}$ and is given by

$$
i_{C E}=\frac{I_{S}}{Q_{B}}\left(\exp \left\{\frac{v_{B E}}{N_{F} \cdot V_{T}}\right\}-\exp \left\{\frac{v_{B C}}{N_{R} \cdot V_{T}}\right\}\right)
$$

where $Q_{B}$ is charge density, $N_{F}$ and $N_{R}$ are forward and reverse current emission coefficients. $V_{T}$ is termed as thermal voltage and is given by $k T / q$. Forward that $i_{C E}=f_{C E}\left(v_{B E}, v_{B C}\right)$, and apply Newton's methodology to voltages and current within the intervals. Then $i_{C E(n+1)}, i_{C E(a)}$ are given by

$$
\begin{aligned}
& i_{C E(n+1)}^{(j+1)}=i_{0(n+1)}^{(j)}+g_{B C(n+1)}^{(j)} v_{B C(n+1)}^{(j+1)}+g_{B E(n+1)}^{(j)} v_{B E(n+1)}^{(j+1)} \\
& i_{C E(a)}^{(j+1)}=i_{0(a)}^{(j)}+g_{B C(a)}^{(j)} v_{B C(a)}^{(j+1)}+g_{B E(a)}^{(j)} v_{B E(A)}^{(j+1)}
\end{aligned}
$$

Parameters $g_{B E(n+1)}^{(j)}, g_{B E(a)}^{(j)}, g_{B C(n+1)}^{(j)}$ and $g_{B C(a)}^{(j)}$ indicate the coefficients of the current sources. These are invariably controlled by the $v_{B E}$. The parameters $i_{0(n+1)}^{(j)}$ and $i_{0(a)}^{(j)}$ are referred as current sources. The results of every parameter are shown as follows:

$$
\begin{gathered}
g_{B C(n+1)}^{(j)}=\frac{I_{S}}{Q_{B(n+1) \cdot N_{F} \cdot V_{T}}} \exp \left\{\frac{v_{B E(n+1)}^{(j)}}{N_{F} \cdot V_{T}}\right\} \\
g_{B C(a)}^{(j)}=\frac{I_{S}}{Q_{B(a)} \cdot N_{F} \cdot V_{T}} \exp \left\{\frac{v_{B E(a)}^{(j)}}{N_{F} \cdot V_{T}}\right\} \\
g_{B E(n+1)}^{(j)}=\frac{I_{S}}{Q_{B(n+1) \cdot N_{R} \cdot V_{T}}} \exp \left\{\frac{v_{B C(n+1)}^{(j)}}{N_{R} \cdot V_{T}}\right\} \\
i_{0(n+1)}^{(j)} \frac{g_{B E(a)}^{(j)}=}{Q_{B(n+1)}}\left(1-\frac{I_{S}}{Q_{B(a)} \cdot N_{R} \cdot V_{T}} \exp \left\{\frac{v_{B C(a)}^{(j)}}{N_{R} \cdot V_{T}}\right\}\right. \\
\left.i_{0(a)}^{(j)}=\frac{I_{S}(n+1)}{Q_{B(a)}}\right)\left(1-\frac{v_{B E(a)}^{(j)}}{N_{F} \cdot V_{T}}\right) \exp \left\{\frac{v_{B C(a)}^{(j)}}{N_{F} \cdot V_{T}}\right\}+\left(-1+\frac{v_{B C(a)}^{(j)}}{N_{R} \cdot V_{T}}\right) \exp \left\{\frac{v_{B C(a)}^{(j)}}{N_{R} \cdot V_{T}}\right\}
\end{gathered}
$$


Hence the computations of RK method of order 4, yield the exact solutions as well as how to diminish or control the error. The error in interpolation can be expressed as

$$
f^{(x)}(t)=2^{x} x ! * O\left(x^{(2+n)}\right), \quad n \rightarrow \infty
$$

approximate values are: $t>h$. Figures 1 to 3 presents the comparison of exact values with RK method of order 4, Heun, Midpoint and Taylors's method with various $h$ values.
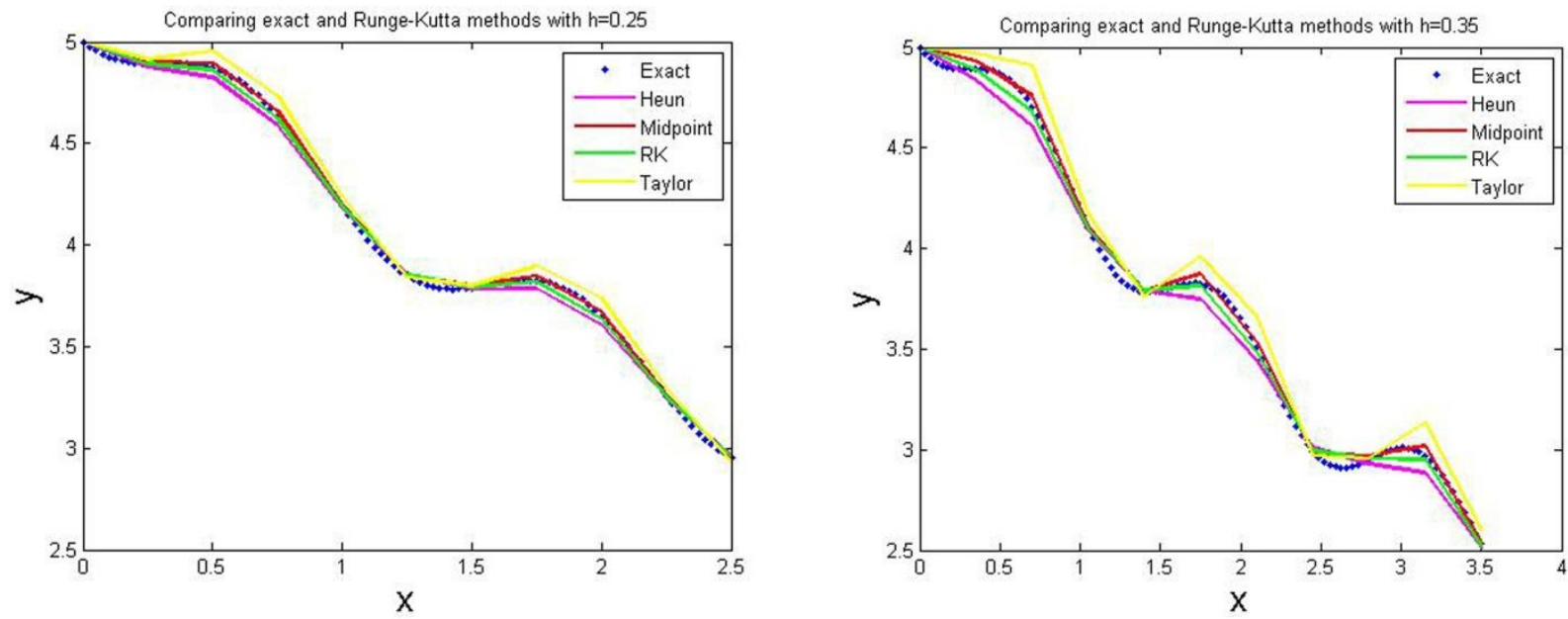

Figure 1: Comparing exact values with RK method of order 4, Heun, Midpoint and Taylors's method when $h=0.25$ and $h=0.35$
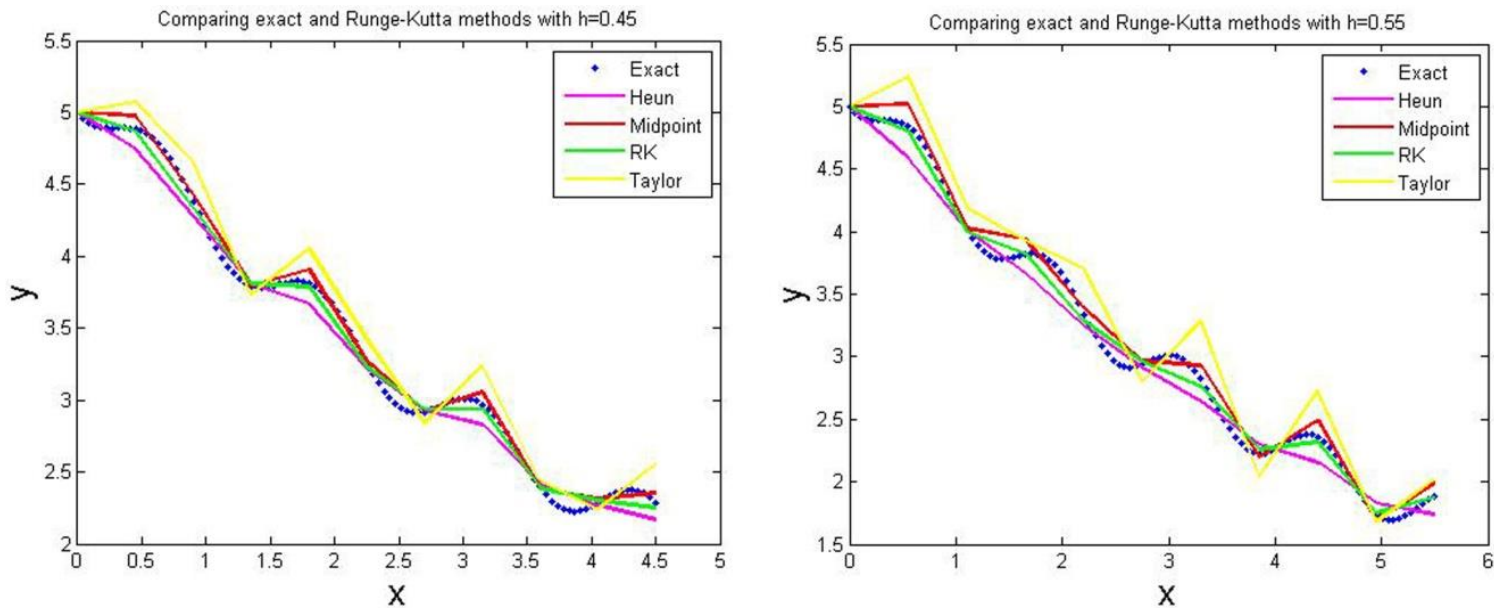

Figure 2: Comparing exact values with RK method of order 4, Heun, Midpoint and Taylors's method when $h=0.45$ and $h=0.55$ 

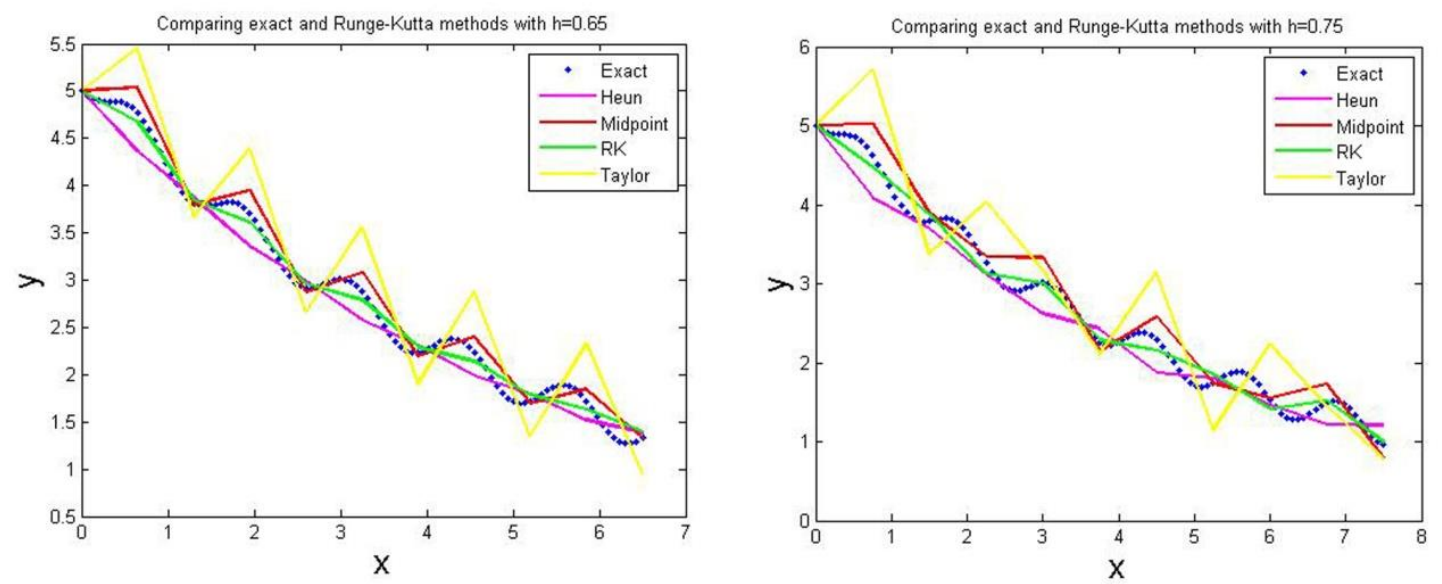

Figure 3: Comparing exact values with RK method of order 4, Heun, Midpoint and Taylors's method when $h=0.65$ and $h=0.75$

\section{Conclusion}

Equilibrium state and array interpretation (Butcher matrix) representation of RK method of order 4 along with the convolution of past, intermediate and present voltages are studied. Constant step size $(h)$ is used until a testing procedure confirms that the discontinuity occurs in the present integration interval. This step size function calculations would take place at the end of the functional calculations, but before the dependent variables were updated. The input parameters indicate the voltage coefficient which is controlled by current sources and measures in a random periodic time. The output parameters provide stable independent values and calculated from past voltage and current values. Implementations of numerical integration for nonlinear capacitors are presented. Finally we compared the exact values with RK method of order 4, Heun, Midpoint and Taylors's method with various $h$ values.

\section{References}

[1] Ahamad N, and Charan, S, Study of numerical solution of fourth-order ordinary differential equations (ODEs) by fifth-order Runge-Kutta method. International Journal of Science, Engineering and Technology, 6, 230-237, 2019.

[2] Alsaedi A, Nieto J.J, Venktesh V, Fractional electrical circuits. Adv. Mech. Eng. 7(12), 1687814015618127,2015

[3] Alexander, Charles K, and Sadiku, Matthew N. O, "Fundamentals of Electric Circuits", 5th Ed, McGraw Hill, Indian Edition, 2013. 
[4] Ashgi, Rizky, Comparison of Numerical Simulation of Epidemiological Model between Euler Method with 4th Order Runge Kutta Method. International Journal of Global Operations Research. 2. 37-44. 10.47194/ijgor.v2i1.67, 2021.

[5] Chauhan V., and Srivastava, P. K. (2018), Trio-Geometric mean-based three-stage Runge Kutta algorithm to solve initial value problem arising in autonomous systems, International Journal of Modeling, Simulation, and Scientific Computing, 9(04), 1850026.

[6] Malarvizhi, M, and Karunanithi, S, 2021, Euler's computational approach for damped oscillatory solutions of RLC circuits, Journal of Physics: Conference Series 1964 (2021) 022007 doi:10.1088/1742-6596/1964/2/022007

[7] Malarvizhi, M, and Karunanithi, S, 2021, Diagonally implicit and explicit Euler's methods for RLC circuit, Turkish Journal of Computer and Mathematics Education Vol.12 No.13 (2021), 1425-1431

[8] Edward Hughes; John Hiley, Keith Brown, Ian McKenzie Smith, "Electrical and Electronic Technology", $10^{\text {th }}$ Ed., Pearson Education Limited, 2008.

[9] Gottlieb, S. (2005). On high order strong stability preserving Runge Kutta and multi step time discretizations. Journal of Scientific Computing, 25(1-2), 105-128.

[10] Hossain, M. J., Alam, M. S., \& Hossain, M. B, A study on numerical solutions of second order initial value problems (IVP) for ordinary differential equations with fourth order and Butchers fifth order Runge Kutta methods. American Journal of Computational and Applied Mathematics, 7(5), 129- 137, 2017.

[11] Islam, M. A. (2015). Accurate solutions of initial value problems for ordinary differential equations with the fourth order Runge Kutta method, Journal of Mathematics Research; Canadian Center of Science and Education, 7(3), 41-45.

[12] Kamruzzama, M, and Nath, M. C, A Comparative study on numerical solution of initial value problem by using Euler's method, modified Euler's method and Runge Kutta method. Journal of Computer and Mathematical Sciences, 9(5), 493-500, 2018.

[13] Kee T A and R. Ranom, Comparison of numerical technique in solving transient analysis of electrical circuits, ARPN Journal of Engineering and Applied Sciences 13(1) (2018) 314-320.

[14] Maffezzoni P, Codecasa, L, and D'Amore, D, Time-domain simulation of nonlinear circuits through implicit Runge-Kutta methods. Institute of Electrical and Electronics Engineers Transactions on Circuits and Systems, 54(2), 391-400, 2007. 
[15] Okten Turac1, M, Ozdemir, M. A sixth-order improved Runge-Kutta direct method for special third-order ordinary differential equations, Numer Methods Partial Differential Eq.. 37: 987-997. https://doi.org/10.1002/num.22562, 2021.

[16] Shruti Tiwari; Ram K. Pandey, Revised version of exponentially fitted pseudo-RungeKutta method, DOI: 10.1504/IJCSM.2021.114199, International Journal of Computing Science and Mathematics, Vol.13 No.2, pp.116 - 125, 2021.

[17] Suhag, A, Transient analysis of electrical circuits using the Runge-Kutta method and its application. International Journal of Scientific and Research Publications, 3, 486490, 2013.

[18] Vijeyata Chauhan and Pankaj Srivastava, Computational Techniques Based on RungeKutta Method of Various Order and Type for Solving Differential Equations. International Journal of Mathematical, Engineering and Management Sciences. Vol 4, No 2, pages 375-386, 10.33889/IJMEMS.2019.4.2-030, 2019.

[19] Yuan H, and Song C, Nonlinear stability and convergence of two step Runge Kutta methods for Volterra delay integro-differential equations. Abstract and Applied Analysis, Article ID 683137, 14 pages, http://dx.doi.org/10.1155/2013/683137, 2013.

[20] You, X, Yao, X and Shu, X. "An Optimized Fourth Order Runge-Kutta Method, Third International Conference on Information and Computing (IEEE), 2010, pp. 46-49, doi: 10.1109/ICIC.2010.195, 2010. 